\title{
Effect of Flask Closure Method and Post-Pressing Time on the Displacement of Maxillary Denture Teeth
}

\author{
Wagner A. Negreiros ${ }^{1}$, Rafael L.X. Consani ${ }^{1, *}$, Marcelo F. Mesquita ${ }^{1}$, Mario A.C. Sinhoreti ${ }^{2}$ and \\ Ivan R. Faria ${ }^{3}$
}

${ }^{I}$ Department of Prosthodontics and Periodontics, Piracicaba Dental School, State University of Campinas, Piracicaba, SP, Brazil; ${ }^{2}$ Department of Restorative Dentistry, Piracicaba Dental School, State University of Campinas, Piracicaba, SP, Brazil and ${ }^{3}$ Department of Dental Materials and Prosthodontics, Araraquara Dental School, Sao Paulo State University, Araraquara, SP, Brazil

\begin{abstract}
The purpose of this study was to verify the influence of flask closure methods and post-pressing times on tooth displacement in maxillary dentures. Twenty similar maxillary dentures were made using a heat-polymerized acrylic resin, and randomly assigned to 4 experimental groups $(n=5)$ according to the protocol: 1-conventional flask closure and immediate polymerization; 2- conventional flask closure and polymerization after 6-hour post-pressing time; 3- RS flask closure system and immediate polymerization; and 4- RS flask closure system and polymerization after 6-hour post-pressing time. Three transverse and 2 anteroposterior linear distances between teeth were measured before and after the denture processing with an optical microscope with accuracy of $0.0005 \mathrm{~mm}$. Analysis of variance (ANOVA) and Tukey's test were performed to compare the results. The 6-hour post-pressing time (conventional $=0.44 \mathrm{~mm}$ and $\mathrm{RS}=0.33 \mathrm{~mm}$ ) showed no statistically significant difference in tooth movement, in relation to the flask closure methods, when compared to the immediate time (conventional $=0.40 \mathrm{~mm}$ and $\mathrm{RS}=0.28 \mathrm{~mm}$ ). The RS system standardized the tooth displacement, independently of the post-pressing times. Dimensional changes were more uniform when the dentures were submitted to the 6-hour post-pressing time and RS flask closure method, suggesting that these factors may reduce the magnitude of tooth movement.
\end{abstract}

Key Words: Denture tooth, flask closure, tooth movement, complete maxillary denture.

\section{INTRODUCTION}

Artificial tooth displacement during complete denture processing changes the harmonious occlusal scheme achieved at the final try-in stage [1]. It is clinically important that dentures have accurate teeth occlusal contacts to obtain normal function. Studies of tooth displacement are important to ensure a more stable occlusal pattern, retention, and functional quality of complete dentures [2].

The processing warpage and base distortion that occur when the polymerized dentures are removed form the cast are considered the major disadvantages of acrylic resin, and these factors can also modify the tooth position [3]. Earlier studies [4,5] showed that the magnitude of dimensional changes is not too great $(-0.1 \%$ to $-0.4 \%)$, and have been reported as having no significant effect on the serviceability of dentures [6]. This undesirable dimensional change remains unresolved [7-11].

A new flask closure system has been proposed to avoid opening the flask during its transference from the hydraulic press to the spring cramp after final closure [8]. Flask opening could allow internal stresses to be released from resin dough and generate undesirable base distortion. Another fact

*Address correspondence to this author at the Faculdade de Odontologia de Piracicaba - UNICAMP, Av. Limeira, 901, 13414-903 - Piracicaba, SP, Brazil; E-mail: rconsani@fop.unicamp.br that may significantly affect occlusal contacts is the delay in polymerizing the denture after the final pressing procedure. A classic study [4] reported that one reason for allowing the flask to stand for at least 1 hour is to allow the resin mass to flow into all regions of the mold and, thus, relieve the internal stress during the early stage after closure. Flask closure methods and post-pressing time variables, however, have not been studied in relation to tooth displacement.

The purpose of this study was to evaluate and to compare the effect of metallic flask closure methods (conventional cramp and RS system) and of post-pressing times before acrylic resin polymerization on the tooth displacement in maxillary complete dentures. The hypothesis tested in this in vitro study was that the tooth displacement could be adversely affected by the flask closure method and postpressing time association.

\section{MATERIAL AND METHODS}

A silicone mold (Elite Double; Zhermack, Rovigo, Italy) was made from a metallic master die simulating an edentulous maxillary arch without irregularities in the alveolar ridge walls. Forty similar stone casts were poured in type III dental stone (Herodent Soli-Hock; Vigodent, RJ, Brazil) in a ratio of $30 \mathrm{~mL}$ water to $100 \mathrm{~g}$ powder.

A uniform denture base was made by the same technician for each cast stone with a $2 \mathrm{~mm}$-thick plate wax (Epoxiglass; 
Epoxiglass Chemical Products, Diadema, SP, Brazil), and measured with a caliper (Golgran; Golgran Dental Products, Sao Paulo, SP, Brazil), for standardization purposes. The height of the occlusion wax rim was $20 \mathrm{~mm}$ in the buccal sulcus of the cast, and $10 \mathrm{~mm}$ in the posterior palate area. The maxillary stone cast was mounted in a Mondial 4000 semiadjustable articulator (Bio-Art Dental Products, Sao Carlos, SP, Brazil) with the wax rim interoclusal relation according to a standardized mandible stone cast with teeth. For this purpose, the references of inter-condylar distance in $\mathrm{M}$, Bennett angle at 15 degrees, and condylar guide at 30 degrees were used.

The arrangement of the left anterior teeth started with the carved wax rim serving as a guide to the positions of the central and lateral incisors and canines. The same procedure was used on the right side. The posterior teeth were arranged starting with the first premolar up to the second molar. The same procedure was used in the right arch. The arrangement of the teeth for the interocclusal relationship was: anterior in vertical overlap and posterior in Angle class I. After arrangement of the first denture, a silicone matrix was made (Zetalabor; Zhermack, Rovigo, Italy) to register the teeth position. The silicone matrix was used as a standard guide for the teeth arrangement in all dentures.

Metallic referential pins (Cadena; Coats Textil Co., Sao Paulo, SP, Brazil) were fixed with instantaneous adhesive (Super Bonder; Loctite, Sao Paulo, SP, Brazil) at the incisal border of the central incisors, buccal cusp of the first premolars, and mesiobuccal cusp of the second molars. The following linear distances were considered: I-I (incisor to incisor), PM-PM (premolar to premolar), M-M (molar to molar), RIRM (right incisor to right molar) and LI-LM (left incisor to left molar). The distances were measured with a STM microscope (Olympus Optical Co., Tokyo, Japan) with an accuracy of $0.0005 \mathrm{~mm}$.

The stone casts were randomly assigned to 4 experimental groups $(n=5)$ : 1 - flask closure with conventional cramp and immediate polymerization; 2- flask closure with conventional cramp and polymerization after a 6-hour post-pressing time; 3- flask closure with the RS system and immediate polymerization; and 4- flask closure with RS the system and polymerization after a 6-hour post-pressing time. The cast and wax pattern sets were flasked in the lower part of traditional brass flasks (Safrany; J Safrany Dental Metallurgy, Sao Paulo, SP, Brazil) with type II dental stone (Pasom; Dental Products, Sao Paulo, SP, Brazil). Petroleum jelly (Labsynth; Labsynth Chemical Products, Diadema, SP, Brazil) was used as a separating medium. After 1 hour, the flasks were placed in boiling water to soften the wax baseplate. The stone was cleaned with liquid detergent (Ype; Chemical Amparo, Amparo, SP, Brazil) and boiling water solution, and two coats of sodium alginate (Isolak; Classico Dental Products, Sao Paulo, SP, Brazil) were used as a mold separator.

Classico polymethyl methacrylate dough (Classico Dental Products, Sao Paulo, SP, Brazil) was used with a monomer: polymer ratio of $1: 3$ by volume. According to the manufacturer, the powder consists of prepolymerized spheres of polymethyl methacrylate and benzoly peroxide as an initiator; and the liquid is an unpolymerized copolymer of methyl methacrylate and ethylacrylate with small amounts of hydroquinone added as an inhibitor. The resin was prepared in accordance with the manufacturer's instructions and each sample was packed in accordance with the group assignments. A plastic sheet was used as a separating medium during the initial flask closure under a load of 850 kilogramsforce (kgf). After this procedure, the plastic sheet was removed and the acrylic resin excess was trimmed.

In the conventional flask closure (Groups 1 and 2), the flasks were placed in traditional cramps after final pressing in a hydraulic press (Linea H; Linea, Sao Paulo, SP, Brazil) under a $1250 \mathrm{kgf}$ load for 5 minutes. The flasks of group 1 were immediately immersed in water at room temperature $\left(25^{\circ} \pm 2^{\circ} \mathrm{C}\right)$ and the acrylic resin polymerized at $74^{\circ} \mathrm{C}$ for 9 hours (temperature rising to $74^{\circ} \mathrm{C}$ at 1 hour, and then maintained at $74^{\circ} \mathrm{C}$ for 8 hours), while the flasks of group 2 were submitted to the same procedures, however the polymerization took place after 6 hours.

In the RS flask closure (Groups 3 and 4), the same trial pack at final closure was accomplished; however, the flask was positioned between the 2 plates of the RS closure system [8]. During the definitive flask closure, the screws of the lower plate were fitted into the holes of the upper plate and, after hydraulic pressure, the screw-nuts were strongly tightened on the screws until just one stop (Fig. 1). Flasks of the Group 3 were immediately immersed in water at room temperature and the acrylic resin polymerized at $74^{\circ} \mathrm{C}$ for 9 hours, while the polymerization of the group 4 took place after 6 hours

After polymerization, the flasks were slowly cooled in the water bath, removed from the thermo-curing unit (Thermotron Dental Products, Piracicaba, SP, Brazil), and bench stored for 3 hours. Afterwards, the dentures were deflasked and the transverse and anteroposterior distances between teeth were measured in conditions similar to those used before denture polymerization. Collected data $(\%)$ were transformed into arc sine of the root of $\mathrm{x} / 100$ and submitted to analysis of variance (ANOVA), considering 2 factors (flask closure and post-pressing time) and their interactions. Differences were submitted to multiple comparison testing (Tukey HSD test at $\alpha=.05$ ).

\section{RESULTS}

In the immediate post-pressing time, the tooth movement was significantly greater for the conventional flask closure method, while there was no difference when the dentures were processed in the 6-hour post-pressing time (Table 1).

Table 1. Means and Standard Deviations of Tooth Displacement (\%) for Flask Closure Method in Relation to the Post-Pressing Time

\begin{tabular}{|c|c|c|}
\hline \multicolumn{3}{|c|}{ Tooth Displacement (\%) } \\
\hline Flask Closure Method & Immediate Time & After 6 Hours \\
\hline \hline Conventional & $0.40(0.05) \mathrm{a}$ & $0.41(0.17) \mathrm{a}$ \\
\hline RS & $0.28(0.12) \mathrm{b}$ & $0.33(0.43) \mathrm{a}$ \\
\hline
\end{tabular}

Means followed by the same lower case letters in each column do not differ significantly at $p<.05$. 
In the conventional flask closure method, there was statistically significant difference in the distance I-I with a greater value for the immediate time (Table 2).

Considering the dentures obtained by the RS flask closure method, no statistically significant difference was observed between the post-pressing times in all distances evaluated (Table $\mathbf{3}$ ).

\section{DISCUSSION}

The hypothesis tested in this in vitro study, that tooth displacement could be adversely affected by the flask closure method and post-pressing time association, was in part accepted. Careful measurements have been taken to overcome denture inaccuracies, such as base distortion and displacement of artificial teeth. These factors are responsible for loss of stability and retention of the dentures, and increase the tooth displacement causing difficulty in the occlusal adjustment procedure. Under clinical conditions, these dimensional changes could also modify the planned vertical occlusion dimension, and cause traumas in mucosa and bone loss [2].

The 6-hour post-pressing time seems to be effective in promoting resin mass relaxation, and probably a possible technical condition to be applied to the laboratory routine. The suggested reason for allowing the flask to stand for some hours before polymerizing is to allow the resin mass to flow into all regions of the mold [4]. In the present study, the 6-hour delayed time showed no statistically significant difference in the tooth movement in relation to the flask closure methods used, when compared to the immediate time (Table 1). Due to resin mass to stay for a longer delayed time before polymerization [4], this procedure probably also reduces the amount of residual monomer present in the resin dough by evaporation [12].
A linear tooth movement occurred in all studied interactions; however, the changes only showed statistically significant difference in the I-I distance (incisor to incisor), when the conventional flask closure was used, demonstrating a higher value for the immediate time (Table 2). A possible explanation for this finding is that the bases were made to be $2 \mathrm{~mm}$ thick and, according to previous studies [13, 14], this factor may reduce the dimensional change of the base.

Other aspects should also be considered: 1- the polymerization shrinkage of the resin is compensated, in part, by the thermal expansion of the resin itself during processing [15]; and 2- the restrictive effect of investing plaster on keeping the teeth in position when the resin induces polymerization and cooling stresses [16]. During or after the procedures, it was also possible that a large amount of internal stresses were relieved before definitive flask closure. Therefore, the remaining internal stresses were not able to promote statistically significant tooth movement after the deflasking denture process.

Previous studies have reported that the largest base dimensional changes are observed in the denture posterior palatal seal $[5,17,18]$, and that a greater magnitude of tooth movement occurs in posterior teeth, altering the occlusal relationship $[19,20]$. However, the present study showed statistically significant tooth movement only in the I-I distance (Table 2). This fact probably occurred due to following technical processing: 1- the mesial space between the incisors was greater during the wax tooth arrangement, allowing them to move toward each other after the flask pressing; 2the placement change occurred in the anteroposterior direction, increasing the transverse distance; and 3- excessive polymerization shrinkage in the anterior region promoted tooth rotation, reducing the I-I distance. The results of this in vitro study are not consistent with the concept that the teeth

Table 2. Means and Standard Deviations of Tooth Displacement (\%) for Dentures Processed by the Conventional Flask Closure Method in Relation to the Post-Pressing Time

\begin{tabular}{|c|c|c|c|c|c|}
\hline \multirow{2}{*}{ Post-Pressing Time } & \multicolumn{5}{|c|}{ Tooth Displacement (\%) } \\
\hline & I-I & PM-PM & M-M & RI-RM & LI-LM \\
\hline Immediate & $1.38(0.43) \mathrm{a}$ & $0.37(0.05) \mathrm{a}$ & $0.22(0.06) \mathrm{a}$ & $0.28(0.12) \mathrm{a}$ & $0.46(0.14) \mathrm{a}$ \\
\hline 6 hours & $0.62(0.20) \mathrm{b}$ & $0.21(0.03) \mathrm{a}$ & $0.21(0.04) \mathrm{a}$ & $0.33(0.06) \mathrm{a}$ & $0.36(0.17) \mathrm{a}$ \\
\hline
\end{tabular}

Means followed by the same lower case letters in each column do not differ significantly at $p<.05$.

Table 3. Means and Standard Deviations of Tooth Displacement (\%) for Dentures Processed by the RS Flask Closure Method in Relation to the Post-Pressing Time

\begin{tabular}{|c|c|c|c|c|c|}
\hline \multirow{2}{*}{ Post-Pressing Time } & \multicolumn{4}{|c|}{ Tooth Displacement (\%) } \\
\cline { 2 - 6 } & I-I & PM-PM & M-M & \multicolumn{2}{|c|}{ RI-RM } \\
\hline \hline Immediate & $0.72(0.37) \mathrm{a}$ & $0.33(0.16) \mathrm{a}$ & $0.26(0.10) \mathrm{a}$ & $0.28(0.08) \mathrm{a}$ & $0.13(0.04) \mathrm{a}$ \\
\hline 6 hours & $1.08(0.32) \mathrm{a}$ & $0.25(0.11) \mathrm{a}$ & $0.25(0.03) \mathrm{a}$ & $0.13(0.04) \mathrm{a}$ & $0.14(0.05) \mathrm{a}$ \\
\hline
\end{tabular}

Means followed by the same lower case letters in each column do not differ significantly at $p<.05$. 
always have a tendency to move toward the midline section of the palate [21]. According to classic studies, the direction of the teeth displacement is complex and it is due to several factors, as internal stress released after polymerization [22], and denture separation from the stone cast $[13,14]$.

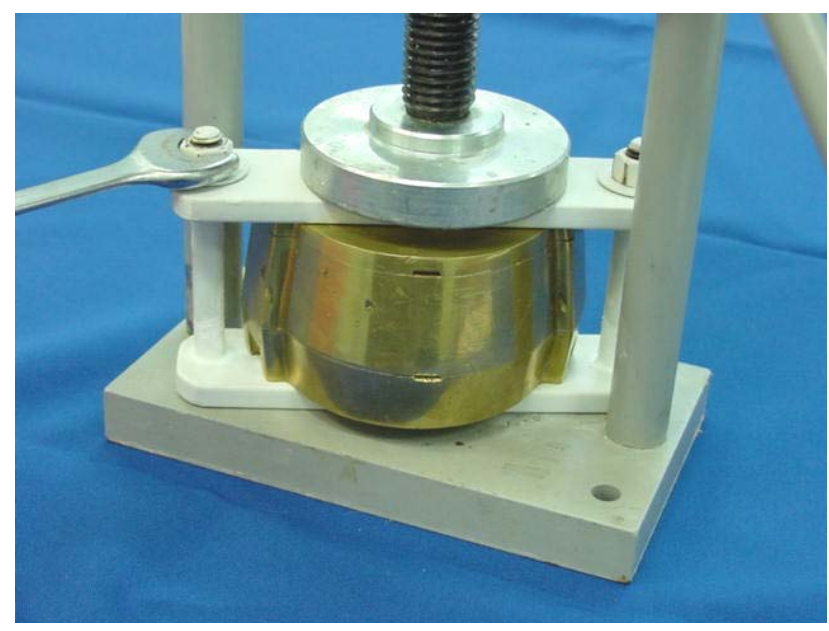

Fig. (1). Flask closure with the RS system.

The RS flask closure method standardized the tooth displacements independently of the post-pressing time used. Table 3 shows the performance of the RS system as an optional packing method to maintain the flask pressure. This system reduced the stress release because flask closure was maintained after press releasing, promoting less distortion in the denture base. Therefore, the reduction of the base inaccuracy allowed the teeth to preserve their position in the denture $[19,23]$.

Reduced dimensional changes in the denture bases processed with the RS flask closure system suggest that the flask closure maintained the acrylic resin dough under constant flask pressure conditions, because the halves remained in contact when the flask was removed from the hydraulic press. This condition may impede or inhibit the premature release of the residual internal stresses from the acrylic resin dough before polymerization [8].

As previous suggested [24], the dimensional changes that occur in acrylic resin dentures remain complex. It is obvious that, independently of the flask closure method and postpressing time used in this study, the complexity of the stresses in the denture also involves other important factors that generate displacement of the artificial teeth.

The results of this study may be of clinical relevance considering that the post-pressing time should be a factor in determining the magnitude of teeth displacement. Although attempts were made to characterize the effect of the postpressing time on tooth movement, this in vitro study is limited in predicting the effect on the changes of the occlusal vertical dimension. Further investigations are necessary to evaluate the effect of the post-pressing time on the mandible denture tooth movement, as well as the effect on the retention and stability of the complete dentures in use.
The clinical relevance of this result can be significant for the comfort of patients, considering that the main purpose of this study was to identify the process that improves the stability of the tooth in the complete dentures.

\section{CONCLUSION}

Within the limitations of this in vitro study, results suggest that the delay of 6 hours before acrylic resin polymerization is an important factor in controlling the magnitude of the tooth movement. The teeth displacement was smaller when the dentures were processed with the RS flask closure system. Delayed time and RS flask closure association has a positive effect on the tooth displacement.

\section{ACKNOWLEDGMENTS}

This study was supported by FUNCAP (Ceara - Brazil) at Piracicaba Dental School, State University of Campinas.

\section{REFERENCES}

[1] Shetty NS, Udani TM. Movement of artificial teeth in waxed trial dentures. J Prosthet Dent 1986; 56(5): 644-8.

[2] McCartney JW. Flange adaptation discrepancy, palatal base distortion, and induced malocclusion caused by processing acrylic resin maxillary complete dentures. J Prosthet Dent 1984; 52(4): 545-53.

[3] Becker CM, Smith DE, Nocholls JI. The comparison of denture base processing techniques. Part 1. Material characteristics. J Prosthet Dent 1977; 37(4): 450-9.

[4] Peyton FA. Packing and pressing denture base resins. J Am Dent Assoc 1950; 40: 520-8.

[5] Firtell DN, Green AJ, Elahi JM. Posterior peripheral seal distortion related to processing temperature. J Prosthet Dent 1981; 45(6): 598-601.

[6] Mowery WE, Burns CL, Dickson G, Sweeney WT. Dimensional stability of denture base resins. J Am Dent Assoc 1958; 57: 345-52.

[7] Huggett R, Zissis A, Harrison A, Dennis A. Dimensional accuracy and stability of acrylic resin denture bases. J Prosthet Dent 1992; 68(4): 634-40.

[8] Consani RLX, Domitti SS, Consani S. Effect of a new tension system used in acrylic resin flasking, on the dimensional stability of denture bases. J Prosthet Dent 2002; 88(3): 285-9.

[9] Boscato N, Consani RLX, Consani S, Cury AADB. Effect of investment material and water immersion time on tooth movement in complete denture. Eur J Prosthodont Rest Dent 2003; 13(4): 164-9.

[10] Pavan S, Arioli-Filho JN, Santos PH, Mollo FA. Effect of microwave treatments on dimensional accuracy of maxillary acrylic resin denture base. Braz Dent J 2005; 16(2): 119-23.

[11] Consani S, Mesquita MF, Nobilo MAA, Henriques GEP. Influence of simulated microwave disinfection on complete denture base adaptation using different flask closure methods. J Prosthet Dent 2007; 97(3): 173-8.

[12] Anusavice KJ. Phillips'science of dental materials. $10^{\text {th }}$ ed. Philadelphia, WB Saunders 1996: 243.

[13] Winkler S, Ortman HR, Morris HF, Plezia RA. Processing changes in complete dentures constructed from pour resins. J Am Dent Assoc 1971; 82: 349-53.

[14] Reeson MG, Jepson NJA. Achieving an even thickness in heatpolymerized acrylic resin denture bases for complete dentures. J Prosthet Dent 1999; 82(3): 359-61.

[15] Kawara M, Komiyama O, Kimoto S, Kobayashi N, Nemoto K. Distortion behavior of heat-activated acrylic denture-base resin in conventional and long, low-temperature processing methods. J Dent Res 1998; 77(6): 1446-53.

[16] Barco MT, Moore BK, Swartz ML, Boone ME, Dykema RW Phillips RW. The effect of relining on the accuracy and stability of maxillary complete dentures - An in vitro and in vivo study. J Prosthet Dent 1979; 42(1): 17-22.

[17] Anthony DH, Peyton FA. Evaluating dimensional accuracy of denture bases with a modified comparator. J Prosthet Dent 1959; 9(4): 683-92. 
[18] Polyzois GL. Improving the adaptation of denture base by anchorage to the casts: a comparative study. Quintessence Int 1990; 21: 185-90.

[19] Wesley RC, Henderson D, Frazier QZ, et al. Processing changes in complete dentures: posterior tooth contacts and pin opening. J Prosthet Dent 1973; 29(1): 46-53.

[20] Consani RLX, Domitti SS, Mesquita MF, Consani S. Influence of flask closure and flask cooling methods on tooth movement in maxillary dentures. J Prosthodont 2006; 15(4): 229-34.
[21] Becker CM, Smith DE, Nicholls BE. The comparison of denturebase processing techniques. Part II. Dimensional changes due to processing. J Prosthet Dent 1977; 37(2): 451-9.

[22] Atkinson HF, Grant AA. An investigation into tooth movement during the packing and polymerization of acrylic resin denture base materials. Austr Dent J 1962; 7(2): 101-8.

[23] Kyes FM. Laboratory's role in successful full dentures. J Prosthet Dent 1951; 1(2): 196-203.

[24] Lorton L, Phillips RW. Heat-released stress in acrylic dentures. J Prosthet Dent 1979; 42(1): 23-6.

Received: June 12, 2008

Revised: October 28, 2008

Accepted: January 21, 2009

(C) Negreiros et al.; Licensee Bentham Open.

This is an open access article licensed under the terms of the Creative Commons Attribution Non-Commercial License (http://creativecommons.org/licenses/ by-nc/3.0/) which permits unrestricted, non-commercial use, distribution and reproduction in any medium, provided the work is properly cited. 